\title{
Probing surface hydrophobicity of individual protein at single-molecule resolution using solid-state nanopores
}

\author{
Ji Li ${ }^{1}$, Zhipeng Tang ${ }^{1}$, Rui Hu${ }^{1}$, Qiang $\mathrm{Fu}^{2}$, Erfu $\mathrm{Yan}^{3}$, Shaoying Wang ${ }^{3}$, Peixuan Guo ${ }^{3}$, \\ Qing Zhao ${ }^{1,4^{*}}$ and Dapeng $\mathrm{Yu}^{1,4^{*}}$
}

Solid-state nanopore is found to be a promising tool to detect proteins and their complexes. Nanopore-protein interaction is a fundamental and ubiquitous process in biology and medical biotechnology. By translocating phi29 connector protein through silicon nitride nanopores, we demonstrate preliminarily probing the surface hydrophobicity of individual protein at single-molecule resolution. The unique "double-level event" observed in the translocation and the ratio of two current drop levels suggest that the position where the interaction occurs is the hydrophobic surface of the protein. We provide a potential method to locate the hydrophobic region of a specific protein surface. This study is of fundamental significance in revealing the important role that hydrophobic interaction plays in nanopore-protein interaction and holds great potential for detecting local surface chemical property of individual protein using solid-state nanopores.

\section{INTRODUCTION}

Nanopores, both biological nanopore [1-5] and solid-state nanopore [6-8], have been developed to be very promising candidates for biological detection at single molecule level, such as DNA [7,9-11], RNA [12,13] and proteins [14-23]. The detection of single molecules is realized through monitoring the temporary ionic current blockage during single molecule translocation through nanopore which is driven by external electric field. By monitoring and statistically analyzing these blockade currents, their durations, and the shapes of the blockades, the properties of biomolecules, such as size, structure, conformation, and dynamic motion can be decoded. The usage of nanopore-based techniques for the detection of biomolecules does not require labeling, surface immobilization, or biomolecular modification. Therefore, nanopore-based techniques are powerful meth- ods for sensing single molecules. Nanopore technique has been shown to be a versatile tool for studying size, charge, or folding state of proteins [14,21,24-26] according to the modulation of ionic current caused by their translocation. Solid-state nanopores have prominent advantages of characterizing single molecules including the versatility of pore dimensions, robustness, longer lifetime, and high chemical resistance to denaturing agents[10,27-34].

Spontaneous adsorption of proteins onto solid-state surfaces [35] is of importance in a broad spectrum of areas, such as the design of nanofluidic devices and functional biomaterials, biosensors, and biomedical molecular diagnosis. Although valuable insight has been put on study of protein adsorption at the liquid-solid interface [23,36-39], this phenomenon is still not comprehensively understood yet. Study of protein adsorption is rather complex due to a multitude of electrostatic and hydrophobic forces among the side chains of the proteins and the reactive groups at the solid-liquid interface [36]. In a sense, solid-state nanopores offer great platforms to study this type of interaction since they can detect protein translocation process at single-molecule level in a nanoscale confined system. Adsorption of single molecule of BSA protein onto $\mathrm{SiN}$ nanopore surfaces has been observed, which was attributed to nonspecific, random, and spontaneous adsorption [37]. Repeated adsorption/desorption events have been observed in protein translocation through the nanotube detention zone [23]. Our understanding of nanopore-protein interaction is still at an early stage and needs further investigation.

The bacterial virus phi29 DNA-packaging nanomotor, with its intricate channel, has inspired applications in nanotechnology $[9,11,40,41]$. It has been demonstrated to have

\footnotetext{
${ }^{1}$ State Key Laboratory for Mesoscopic Physics, School of Physics, Peking University, Beijing 100871, China

${ }^{2}$ Beijing Research Institute of Chemical Industry, Beijing 100013, China

${ }^{3}$ Nanobiotechnology Center, Markey Cancer Center, and Department of Pharmaceutical Sciences, University of Kentucky, Lexington, KY 40536, United States

${ }^{4}$ Collaborative Innovation Center of Quantum Matter, Beijing 100871, China

*Corresponding authors (emails: zhaoqing@pku.edu.cn (Zhao Q); yudp@pku.edu.cn (Yu D))
} 
the capability to detect single-stranded DNA (ssDNA) and double-stranded DNA (dsDNA) at single-molecule level by inserting into planar lipid bilayers with an appropriate size ion channel for single DNA translocation [11]. The protein hub of this motor is a truncated cone structure, termed phi29 connector protein (Figs 1a and b), which allows double-stranded DNA (dsDNA) to enter during maturation and exit during infection. The protein has different chemical characteristic along its surface: the two terminal parts are hydrophilic while the middle part is hydrophobic (Fig. 1a). This peculiar surface chemical property inspires us to study the protein-nanopore interaction since hydrophilic and hydrophobic region may result in different interaction with the solid surfaces.

In this work, by studying the translocation behavior of the phi29 connector protein through SiN nanopores, we successfully probed the surface hydrophobicity of the detected molecule at single-molecule resolution. The observed unique "double-level" translocation events can be explained by the hydrophobic interaction between the hydrophobic part of the protein and the SiN nanopore surface. The ratio of the two current drop levels of such "double-level event" indicates that it is the hydrophobic part of the protein where the interaction happens. To some extent, we provide a potential method to locate the hydrophobic region of a specific protein surface. Additionally, "anomalous" translocation of phi29 connector protein has been observed at $\mathrm{pH}$ 9. By comparison of measurement at $\mathrm{pH}$ 4, we attribute such "anomalous" translocation to the electroosmosis dominant protein transport. Our study is of fundamental significance in revealing the important role that hydrophobic interaction plays in nanopore-protein interaction. Solid-state nanopore provides unique platforms to study the interaction between protein and solid surfaces
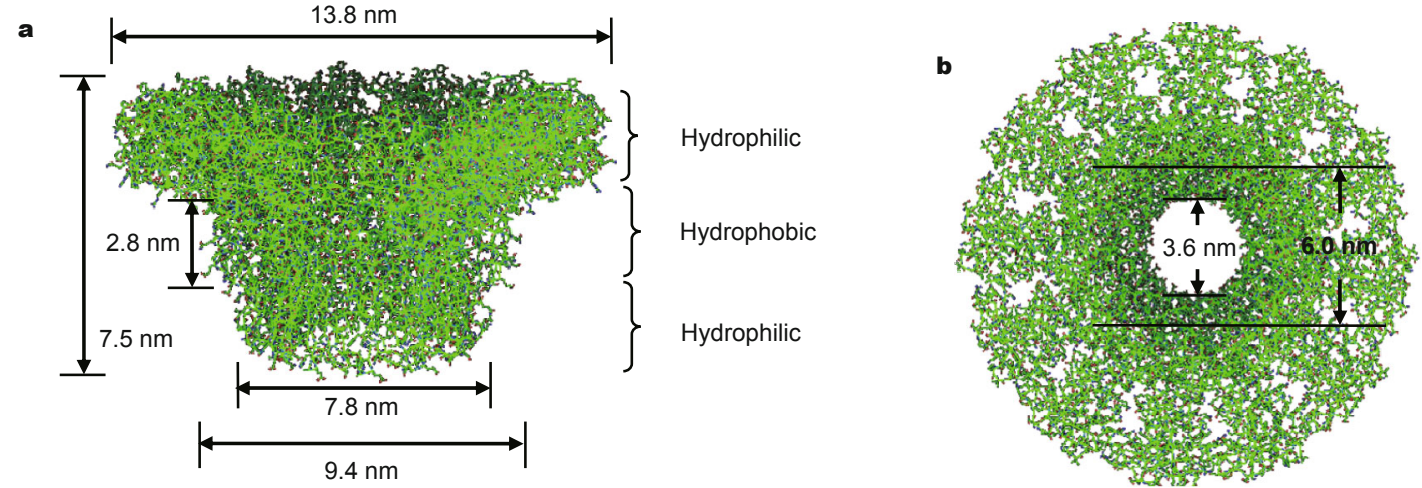

c

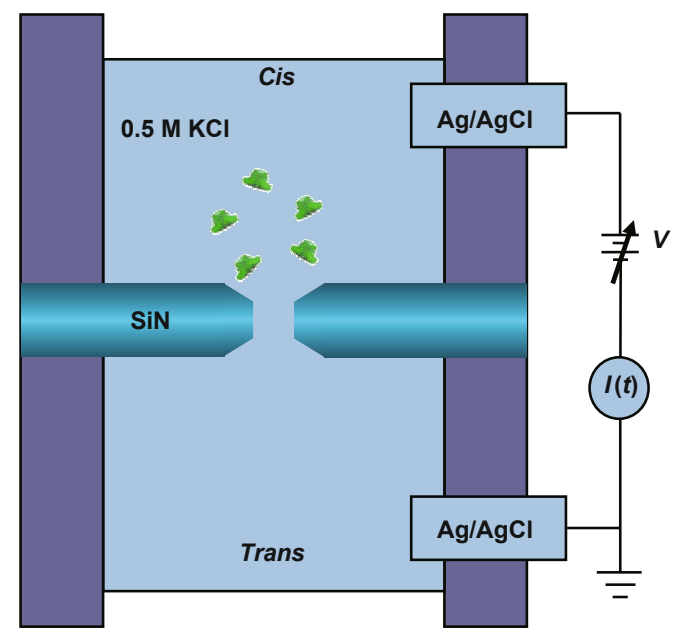

Figure 1 (a) Side view of the phi29 connector protein showing the hydrophobic part, hydrophilic part and the diameter of the channel. "Big head" of the protein is the $\mathrm{C}$ terminal and "small head" is the $\mathrm{N}$ terminal. (b) Top view of the protein showing the diameter of the narrow part and wide part of the channel. (c) Schematic diagram of phi29 connector protein translocation experiment through SiN nanopore. 
at single-molecular level.

\section{RESULTS AND DISCUSSION}

Fig. 1c shows the schematic diagram of the experimental configuration of the objective protein molecules (phi29 connector protein) translocation through a SiN nanopore. Generally, we fabricated a single nanopore in a freestanding membrane of $80 \mathrm{~nm}$ thick low-stress SiN membrane using the focused electron beam of a transmission electron microscope (TEM). The nanopore chip sealed with polydimethylsiloxane (PDMS) gaskets is mounted between two polyether ether ketone (PEEK) chambers. The trans and cis chambers full of ionic solution and electrically contacted with $\mathrm{Ag} / \mathrm{AgCl}$ electrodes are isolated by the chip so that the single SiN nanopore is the only transmembrane channel connecting these two parts. Charged phi29 connector proteins can be driven through the nanopore when a bias voltage is applied across the membrane. Change of the ionic current with time is monitored, implying the translocation of individual phi29 connector protein. The protein contains 12 copies of gp10 subunits, each having $3 \alpha$-helixes, to form a central channel. The diameter of the widest and nar-
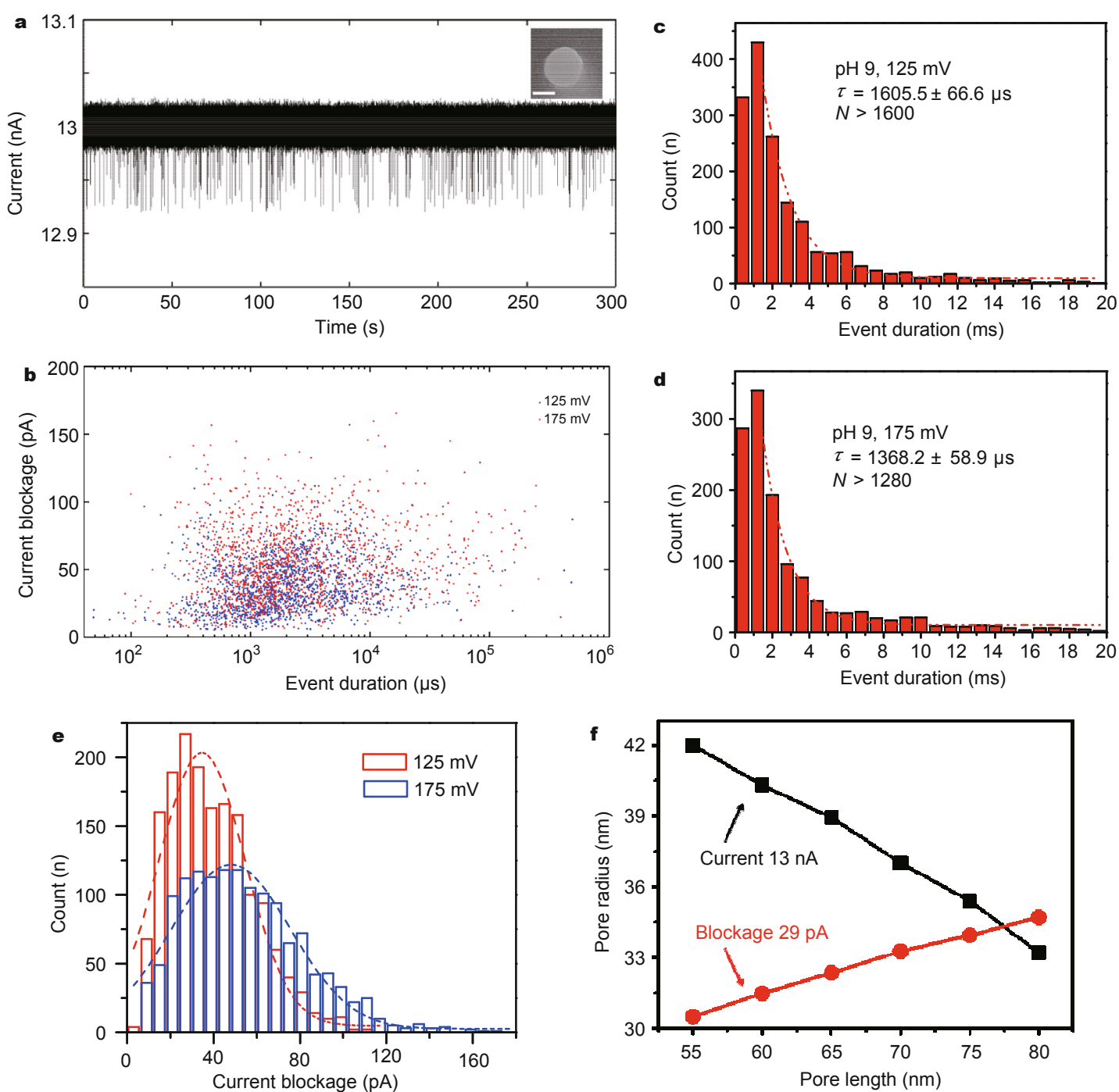

Figure 2 (a) Current trace of phi29 connector protein translocation measured under $100 \mathrm{mV}(0.5 \mathrm{M} \mathrm{KCl}, 5 \mathrm{mM}$ Tris, pH 9), presenting stable and smooth trace. Inset: TEM image of the used SiN nanopore. Scale bar: $40 \mathrm{~nm}$. (b) Scatter plot of current blockage vs. event duration of phi29 connector protein translocation under voltage of $125 \mathrm{mV}$ (blue, $N=1683$ ) and $175 \mathrm{mV}$ (red, $N=1401$ ). (c, d) Histogram of event duration of Fig. $2 \mathrm{~b}$ showing the exponential tail fit. Characteristic event duration, $\tau$, is defined by an exponential decay fit to the tail of the event duration histogram. (e) Distribution of current blockage of Fig. 2b showing the most probable current blockage is in direct proportion to the applied voltage. (f) Calculated open current of 13 $\mathrm{nA}$ (black square) and normalized current blockage of $29 \mathrm{pA}$ (red circle) as a function of nanopore radius and nanopore length. 
rowest channel is $6 \mathrm{~nm}$ and $3.6 \mathrm{~nm}$, respectively (Fig. 1b).

Fig. 2a shows a typical current trace after phi29 connector protein is added to the cis reservoir $(\mathrm{pH} 9,0.5 \mathrm{M}$ $\mathrm{KCl}, 5 \mathrm{mM}$ Tris), presenting a stable and smooth trace with high throughput. We call each temporary drop in ionic current, which is attributed to a single phi29 connector protein translocation through the pore, "a translocation event". Each translocation event can be characterized by two parameters: current blockage and event duration. Fig. $2 \mathrm{~b}$ shows the scatter plot of current blockage $v s$. event duration of phi29 connector protein translocation under two different voltages. Event durations mainly fall between 1 and $10 \mathrm{~ms}$, implying that phi29 connector protein has interaction with the hydrophobic surface of SiN membrane during translocation. Event duration of protein translocation in previous work was usually within several hundreds of microseconds, resulting in a limited temporal resolution hindering the characterization of many biomolecules $[16,42,43]$. Fologea et al. [16] successfully probed BSA molecules using SiN nanopores, which has similar molecular weight as phi29 connector protein, with most probable translocation time of nearly $110 \mu \mathrm{s}(0.4 \mathrm{M} \mathrm{KCl}, 120$ $\mathrm{mV}, 16 \mathrm{~nm}$ diameter nanopore). Events with such short translocation time could be reasonably recognized as free translocations with no strong interaction between the protein molecule and the nanopore, which was also observed in our experiments. However, we also observed that more than $70 \%$ events had a translocation time more than $1 \mathrm{~ms}$ (both at $125 \mathrm{mV}$ and $175 \mathrm{mV}$ in Fig. 2b), implying strong interaction between the protein and the nanopore. The interaction dominated long-lived protein translocations have high temporal resolution and bear great potential to sense more specific structure of protein molecules. Histogram of event duration (Figs $2 \mathrm{c}$ and $\mathrm{d}$ ) shows the mono exponential tail fit, indicating the presence of the protein-pore interaction [44]. Characteristic event duration, $\tau$, defined by an exponential decay fit to the tail of the event duration histogram, declines as the bias voltage rises. Fig. 2e shows the Gaussion distribution of the current blockage under 125 (red) and $175 \mathrm{mV}$ (blue). Mean current blockages are 36 and $49 \mathrm{pA}$, respectively, in direct proportion to the applied voltage, revealing similar behavior of DNA translocation where current blockage increases linearly with the applied voltage [45].

Several evidences demonstrate that the current blockage signal reveals a real translocation process of phi29 connector protein through nanopores. Firstly, linear increase of current blockage as a function of applied voltage (Fig. $2 \mathrm{e}$ ) is a strong evidence for real translocation behavior. Secondly, Poisson-Boltzmann formulation was used to cal- culate the openpore current and DNA translocation blockage in nanopores in our previous work [46]. By setting the DNA radius as $1.25 \mathrm{~nm}$, due to the Stern layer effect on DNA solution interface, and associated with other original boundary condition setting, the pore-geometry-variation curves based on a specific openpore current or DNA blockage can be independently mapped. The crossing point of those two curves can determine the pore geometry (pore radius and pore length) at a certain openpore current and DNA blockage [31]. Here, by applying the Poisson-Boltzmann formulation and setting the original boundary conditions, we realize the simulation of pore-geometry-variation curves based on the specific openpore current and protein translocation blockage (Fig. 2f). In our experiment under $100 \mathrm{mV}$, the openpore current is $13 \mathrm{nA}$ and the mean protein blockage is about $29 \mathrm{pA}$. We can get a pore radius of $34.5 \mathrm{~nm}$ by obtaining these two curves then finding the point of intersection, which is in agreement with the value $33 \mathrm{~nm}$ obtained from TEM figures (Fig. 2a inset). Thirdly, the current blockage can be used to estimate the volume of protein. Protein diameter can be estimated from the current blockage data based on [47]

$$
d_{\text {pro }}^{3}=s \frac{\Delta I}{I_{0}}\left(l_{\text {pore }}+0.8 d_{\text {pore }}\right) d_{\text {pore }}^{2}
$$

where $d_{\text {pro }}$ is the equivalent protein diameter, $d_{\text {pore }}$ is the pore diameter, $l_{\text {pore }}$ is the pore length, $\Delta I$ is the statistical mean current blockage and $I_{0}$ is the mean openpore current. According to the Poisson-Boltzmann calculation based on the translocation experimental data, the calculated protein volume falls in a range between 483 and 642 $\mathrm{nm}^{3}$ by considering that the phi2 9 connector protein has a spherical geometry. The total volume of phi29 connector protein $\left(560 \mathrm{~nm}^{3}\right)$, according to simple calculation of Fig. $5 \mathrm{a}$, fits well within the range calculated from conductance and current blockage, which is another strong evidence for real translocation of protein through nanopores.

During analyzing translocation event signal of phi29 connector protein, one kind of anomalous events have been observed (Fig. 3a). Such events have two different current blockage levels, $I_{1}$ and $I_{2}$, and two time durations, $t_{1}$ and $t_{2}$, correspondingly. We call such signals as "double-level events" compared to the normal "single-level events". These double-level events cannot resemble the partially folded events in DNA translocation (Fig. 3b) since the protein will be stable in our solution surroundings without folding. Additionally, double-level events always show a shallower blockage $I_{1}$ first followed by a deeper blockage $I_{2}$, which is different from the partially folded events of DNA translocation who has a deeper blockage first (Fig. 3b). We 
a

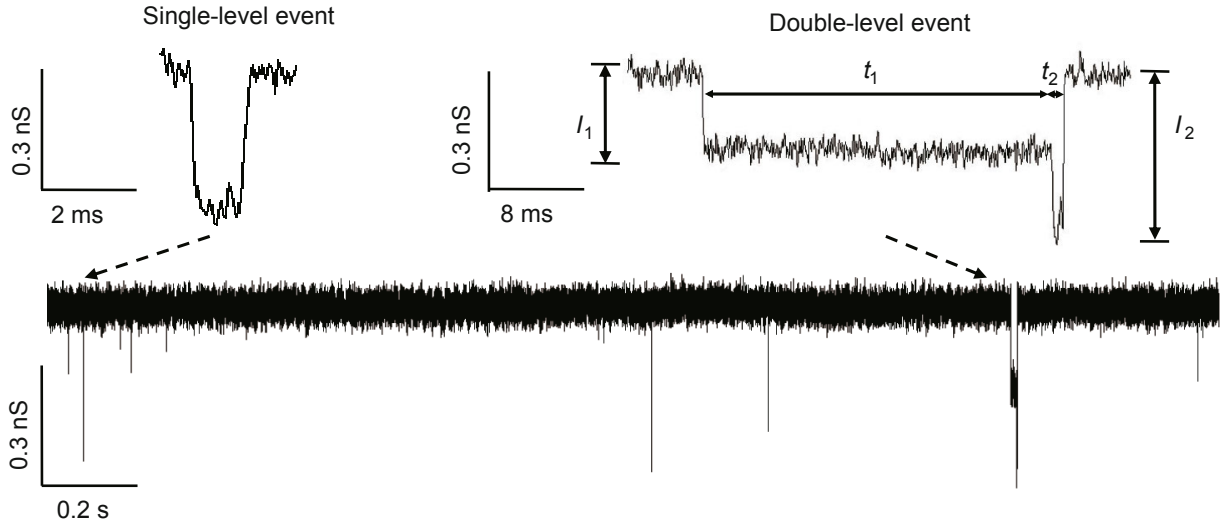

b

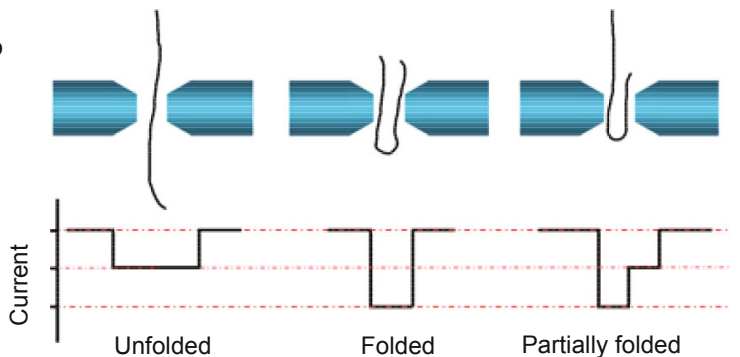

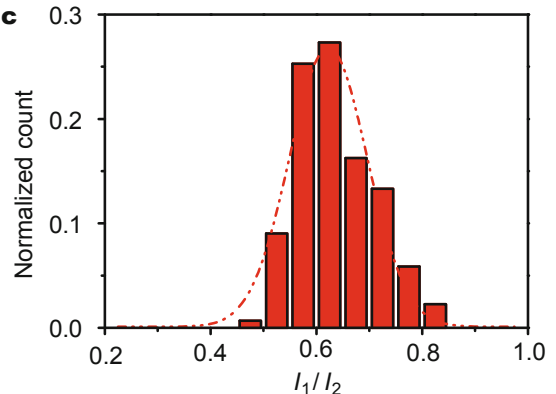

Figure 3 (a) Current trace showing two types of typical events of phi29 connector protein translocation: single-level event (left) and double-level event (right). (b) Three types of typical translocation events of dsDNA through SiN nanopores. (c) Histogram of $I_{1} / I_{2}$ about all 160 detected double-level events in Fig. 2b (125mV: 94 and 175mV: 66) showing that most of the ratios fall into 0.5 to 0.8 .

also found that the ratio of $I_{1}$ and $I_{2}$ falled into a certain range. $90 \%$ of the $I_{1} / I_{2}$ values of all detected double-level events fall within the range from 0.55 to 0.84 , as shown in the distribution plot of Fig. 3c. Another three parallel experiments show similar findings of "double-level events" and the certain range of $I_{1} / I_{2}$ (Fig. 4). Over $90 \%$ of the $I_{1} /$ $I_{2}$ values fall into the range of 0.55 to 0.84 in each experiment, as shown in Fig. 4 . The certain value of $I_{1} / I_{2}$ suggests that it may be the certain region of protein molecules that interacts with nanopore wall. It should be noted that the "double-level events" only account for a small portion of the total detected events: for the three parallel experiments, the number of "double-level events" is 215,443 and 651, respectively, with the total events number of $\sim 3500, \sim 7000$ and $\sim 10,000$, respectively.

Protein adsorption is a complex process involving van der Waals, hydrogen bonding, hydrophobic interaction and electrostatic interaction. Surface-protein interaction has been proved to be affected by underlying surface chemical property, structural information of protein surface and surface topography, among which surface chemical property has been shown to play a fundamental role in protein adsorption. From Fig. 1a, three segments of phi29 connec- tor protein have different surface chemical properties, with two segments showing hydrophilic property and the middle segment showing hydrophobic property. The unique surface chemical property inspires us to probe the role it plays in the interaction between protein and the hydrophobic nanopore surface. Roach et al. $[38,39]$ reported that proteins had a much higher binding affinity toward hydrophobic rather than hydrophilic surface. They implied that it was the hydrophobic region rather than the hydrophilic region of protein surface that contributed to surface-protein interaction, which can be explained as hydrophobic interaction. Then hydrophobic interaction possibly accounts for the adsorption (or interaction) between protein and inorganic surface ( $\mathrm{SiN}$ ), therefore, the hydrophobic region of the phi29 connector protein may prefer to interact with the hydrophobic SiN nanopore surface.

Next we try to explain why the "double-level event" shows a shallower blockage first followed by a deeper blockage and associates with hydrophobic interaction. Fig. $5 \mathrm{a}$ is the equivalent schematic showing the geometrical structure of phi29 connector protein. It can be regarded as three segments of cylinder with different inner and outer diameters as well as different surface chemical properties. 

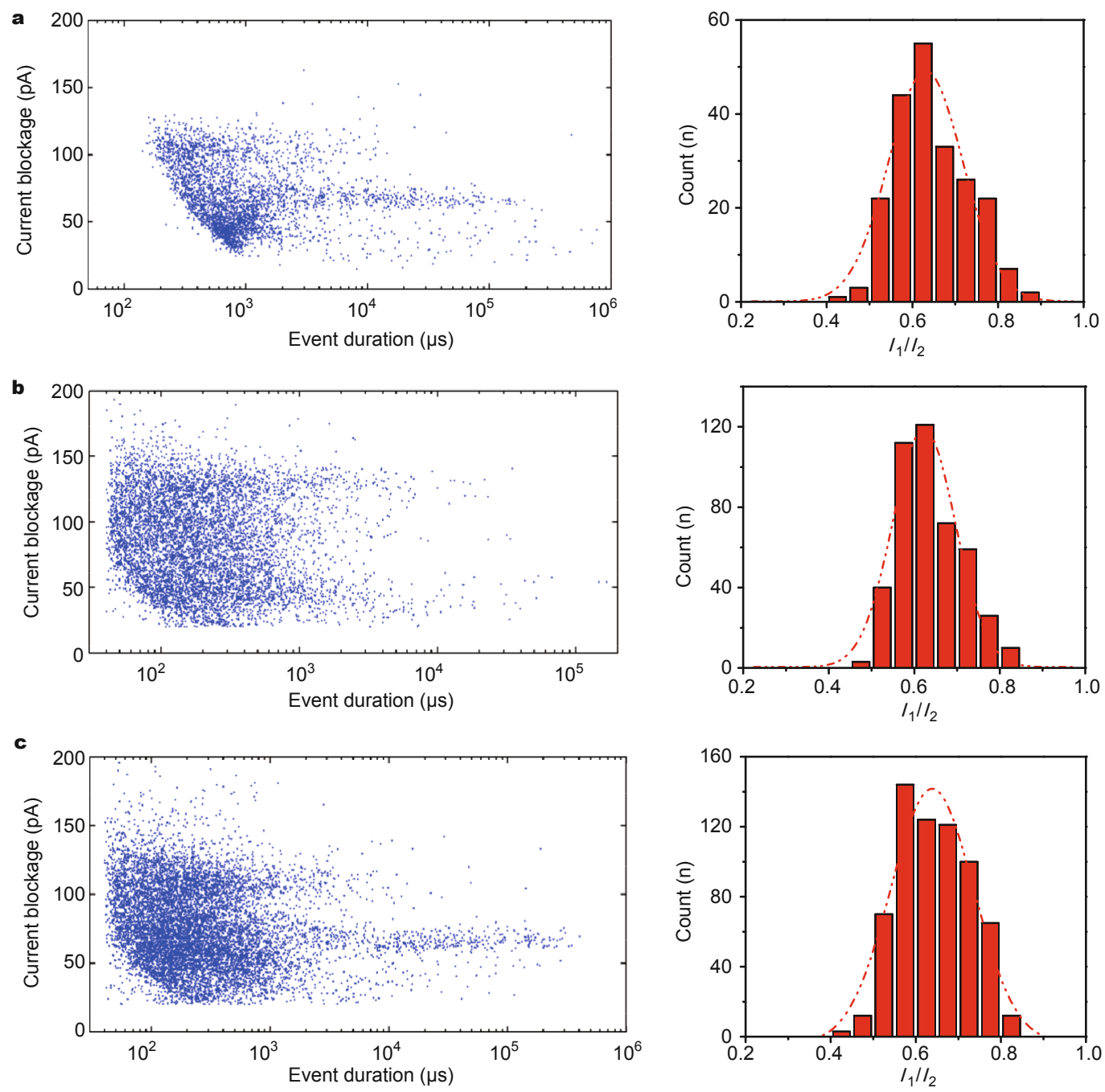

Figure 4 Another three experiments of phi29 connector protein translocation at $\mathrm{pH} 9$ under voltage of $100 \mathrm{mV}$ with nanopore radius of $21-23 \mathrm{~nm}$. (a, b, c left) Scatter plot of current blockage $v s$. event duration of phi29 connector protein translocation events (with total number more than 3500 , 7000 and 10,000 respectively). (a, b, c right) Histogram of $I_{1} / I_{2}$ about detected double-level events (with number of 215 , 443 and 651 , respectively) showing excellent accordance to Fig. $3 \mathrm{c}$ with more than $90 \%$ falls into 0.5 to 0.8 .

The volume of the three segments of phi29 connector protein from top to bottom (Fig. 5a) are $V_{1}, V_{2}, V_{3}$, respectively, and the middle part has hydrophobic surface while the top and bottom parts are hydrophilic. As is only $7.5 \mathrm{~nm}$ in length, the protein cannot fulfill the entire SiN nanopore along the longitudinal direction, compared to DNA translocation, since the effective nanopore thickness is usually more than $40 \mathrm{~nm}$ in our experiment. Considering that the blockage is in proportional to the nanopore volume occupied by the protein, therefore, a shallower current blockage is observed if the protein is partly inside the nanopore when the interaction occurs as shown in Figs $5 b$ and c. The shallower current blockage $I_{1}$ (Fig. 3a) can be interpreted as phi29 connector protein interacting with nanopore wall for a certain time $\left(t_{1}\right.$ in Fig. 3a) with only part of its volume inside the nanopore. Then deeper $I_{2}$ is caused by desorption of protein from nanopore wall with its entire volume inside the nanopore (Fig. 5d).

The certain value of $I_{1} / I_{2}$ inspires us to investigate the certain region that the hydrophobic interaction occurs. From the dimension shown in Fig. 5a, we can get $V_{1}=$ $305.5 \mathrm{~nm}^{3}, V_{2}=165.7 \mathrm{~nm}^{3}, V_{3}=88.6 \mathrm{~nm}^{3}$, and total $V=$ $V_{1}+V_{2}+V_{3}=559.8 \mathrm{~nm}^{3}$, respectively. Assuming that $\mathrm{C}$ terminal (big head) of phi29 connector protein moves in the front and considering that the interaction site is among the hydrophobic region $V_{2}, I_{1}$ should be caused by $V_{1}$ at least, 
a

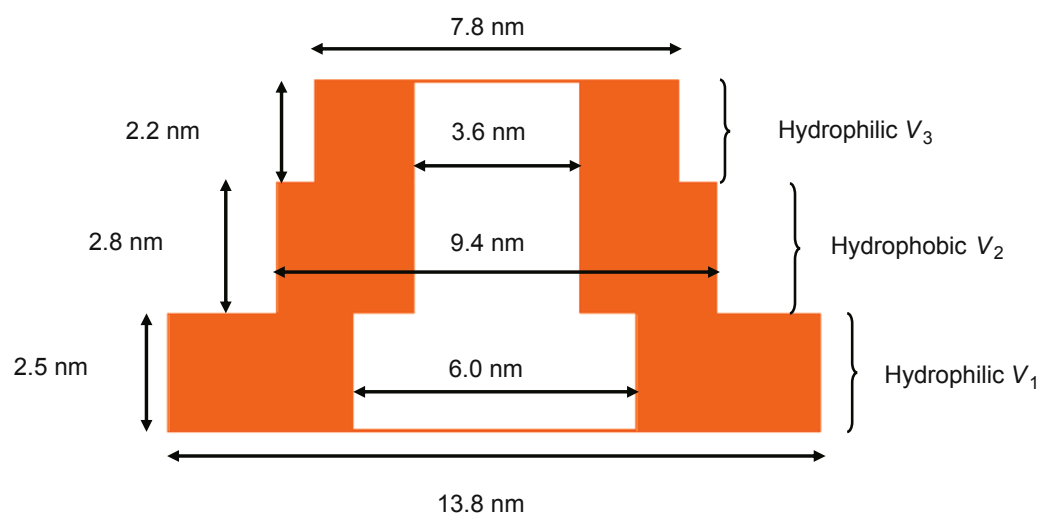

b

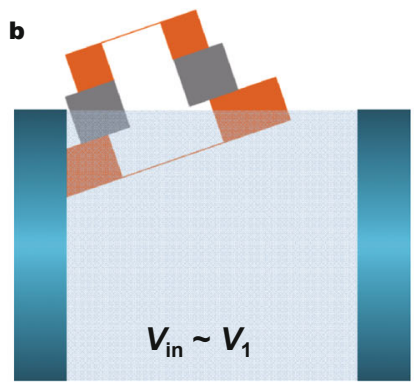

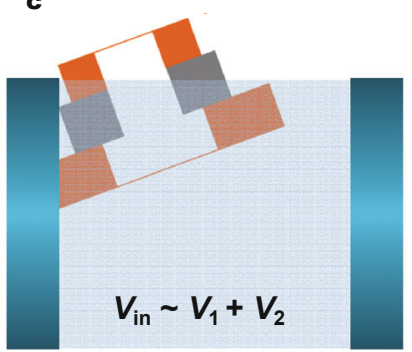

d

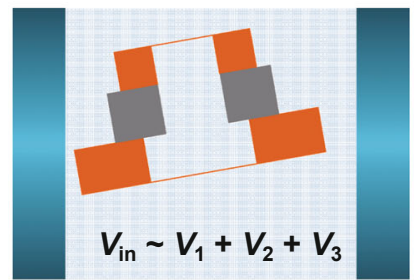

Figure 5 (a) Equivalent schematic diagram of phi29 connector protein showing three segments with corresponding channel sizes and different surface properties. (b-d) Schematic illustration indicates the location where double-level events might occur. (b, c) The adsorption of protein occurs as part of the phi29 connector protein is inside nanopore and part is outside of nanopore. The interaction occurs at the hydrophobic part of protein, as indicated by grey color. (d) After desorption, during translocation, protein has its entire volume inside the nanopore.

and $V_{1}+V_{2}$ at most, which is in the range of $V_{1} \sim V_{1}+V_{2}$, as shown in Figs $5 \mathrm{~b}$ and c. $I_{2}$ is caused by the entire $V$, which is $V_{1}+V_{2}+V_{3}$, as shown in Fig. $5 \mathrm{~d}$. Thus the value of $I_{1} / I_{2}$ should be in the range of $V_{1} / V$ and $\left(V_{1}+V_{2}\right) / V$. The calculation results of $I_{1} / I_{2}$ give a range from 0.55 to 0.84 , revealing good accordance with the experimental value of $I_{1} / I_{2}$ (Figs $3 c$ and 4 ), in which the value is mainly distributed between 0.5 and $0.8 . I_{1} / I_{2}$ should not present such value if the interaction occurs at location in $V_{1}$ or $V_{3}$. Therefore, the observed anomalous "double-level events" can be reasonably attributed to the hydrophobic interaction, and the interaction occurs at, or at least, near the hydrophobic region (middle part) of phi29 connector protein. It is essential to make it clear that the double-level events are only part of the interaction-dominated events. If the interaction happens when the protein is totally inside the nanopore, events only present long translocation time without "double-level" shape, as supported by our data in Figs 2 b and 4 . As the protein size is much smaller than the pore, the small amount of double-level events (5.2\% of all the events) is reasonable as there is big probability that the protein interacts with the pore totally inside. To some extent, we offer a promising method to probe the surface hydropho- bicity of a specific protein on its surface region during its translocation through solid-state nanopores, which holds great potential on more accurate detection of local surface physical or biochemical modification of proteins and other biomolecules with solid-state nanopores. Further study of the orientation of protein translocation and more specific location will be conducted.

We also observed "anomalous" translocation of phi29 connector protein through $\mathrm{SiN}$ nanopores at $\mathrm{pH}$ 9: negatively charged proteins crossed the pore toward the negatively charged electrode. The net charge of phi29 connector protein as a function of $\mathrm{pH}$ is shown in Fig. $6 \mathrm{a}$ by calculation according to the $\mathrm{pKa}$ of every amino acid of the protein. At $\mathrm{pH} 9$, the entire phi29 connector protein is negatively charged. We observed that the negatively charged phi29 connector proteins passed across the nanopore from the positively charged electrode toward the negatively charged one at $\mathrm{pH}$ 9. As the electric force exerted on the protein is toward the cis side where we added the sample, there should be some other force or effect that pushes phi29 connector protein passing through the nanopore from cis to trans side. In fact, such "anomalous" translocation across $\mathrm{SiN}$ nanopore was reported in previous work and the elec- 
troosmotic (EO) effects were proved to play a vital role in these translocations [48-50]. EO effect may enhance or counteract electrophoresis and it is the combined action of electrophoretic (EP) and EO forces that governs the translocation direction of protein across the nanopores [48].

The protein and wall of $\mathrm{SiN}$ nanopore is negatively charged on the whole at $\mathrm{pH} 9$ so the electrophoretic transport is toward the cis side while the EO transport is toward the trans side. The observed "anomalous" translocation indicates that EO effect overwhelms electrophoretic effect at $\mathrm{pH} 9$ (Fig. 6b). Phi29 connector protein contains only -11.46 unit charges at $\mathrm{pH} 9$, which are nearly negligible compared to that of DNA with the same molecular weight, so the electric force on the protein is several orders smaller. Meanwhile, phi29 connector protein is more inclined to be pushed forward by the axial force of EO flow while linear DNA is mainly affected by the tangential force of EO flow. That may qualitatively account for why such "anomalous" translocation is mostly observed in protein translocation. The disappearance of any translocation signal at the reverse voltage (cis side: electrically grounded, trans side: $100 \mathrm{mV}$ ) also proves this speculation. As the EO flow exists near the nanopore surface, it will exert bigger force on the protein during its translocation through smaller pores. Therefore the translocation time will be generally shorter. That could help to explain the smaller portion of the interaction-dominated events in Fig. 4 compared to Fig. 2 b.

We also conducted the translocation experiments at $\mathrm{pH} 4$ when phi29 is positively charged to further confirm whether the explanation of EO flow was reasonable in determining the translocation direction. At $\mathrm{pH} 4$, only when negative potential was applied to the trans side did we observe translocation signals in the current traces which signified phi29 connector proteins passing through nanopore. At $\mathrm{pH} 4$, the protein is positively charged (Fig. 6a) and EO flow can be negligible since the SiN nanopore is nearly electro neutral on the wall at this $\mathrm{pH}$ [51]. So translocation can only be observed when the negative potential is applied to trans side as the electrophoretic force drives the protein to pass through. The "normal" translocation is observed at $\mathrm{pH} 4$ at the absence of EO flow, which confirms our explanation of the "anomalous" translocation at $\mathrm{pH}$ 9. Fig. $6 \mathrm{c}$ shows the scatter plot of current blockage vs. event duration at $V=100$ (red) and $150 \mathrm{mV}$ (blue) at $\mathrm{pH}$ 4. Due to
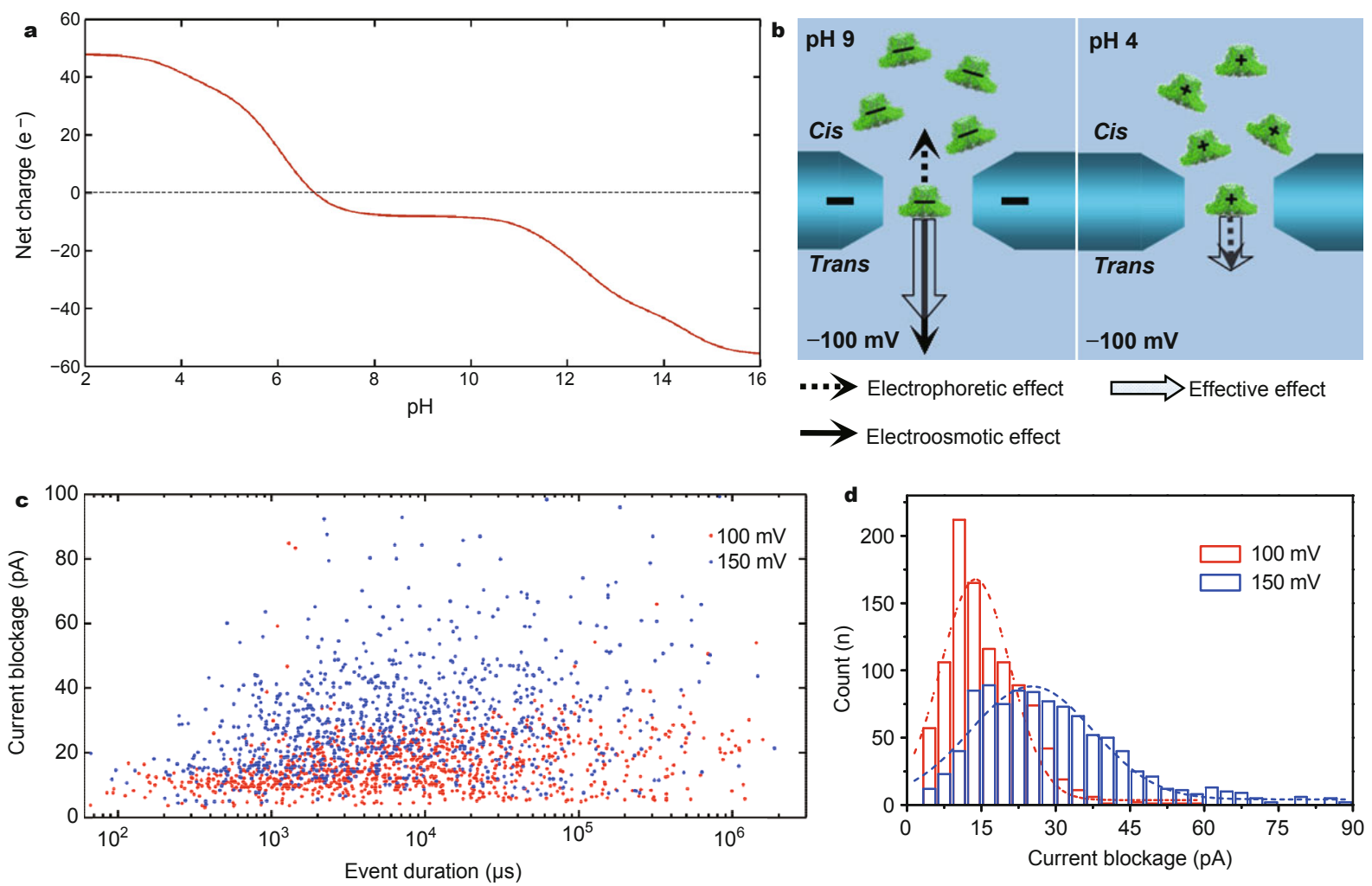

Figure 6 (a) Calculated net charge of phi29 connector protein as a function of pH. (b) Schematic illustration of phi29 connector protein translocation experiments at $\mathrm{pH} 9$ and $\mathrm{pH} 4$. Arrows represent electroosmotic and electrophoretic effects, and arrow length represents only relative value. (c) Scatter plot of phi2 9 connector protein translocation events at $V=100 \mathrm{mV}$ (red) and $150 \mathrm{mV}$ (blue), pH 4, $0.5 \mathrm{M} \mathrm{KCl,} 5 \mathrm{mM}$ Tris. (d) Distribution of current blockage at $V=100 \mathrm{mV}$ (red) and $150 \mathrm{mV}$ (blue). 
the absence of EO flow at pH 4, small electrophoretic force makes the translocation slower compared to that at $\mathrm{pH} 9$, and several event durations nearly reach 10 seconds. The most probable current blockage shown by Gaussion fitting (Fig. 6d) is proportional to the applied voltage, which agrees with what has been analyzed at $\mathrm{pH} 9$.

\section{CONCLUSION}

In conclusion, the observed unique "double-level event" in translocating single phi29 connector protein molecules through SiN nanopores reveals the presence of protein-nanopore interaction. The certain value of $I_{1} / I_{2}$ indicates that hydrophobic interaction between protein and nanopore occurs in the region of hydrophobic surface of the protein molecule. Meanwhile, we observed electroosmotic dominant protein transport at $\mathrm{pH} 9$. In a word, we provide a potential method of precisely positioning the surface hydrophobic region of a specific protein inside nanopore at single-molecular resolution. Our work offers an insight into probing surface chemical property of proteins and provides a platform to study the protein-nanopore interaction.

\section{EXPERIMENTAL SECTION}

\section{Nanopore fabrication}

$2 \mu \mathrm{m}$ thick silicon oxide buffer layer and $80 \mathrm{~nm}$ silicon nitride were deposited on both sides of a $400 \mu \mathrm{m}$ thick $\langle 100\rangle$ Si wafer. A $20 \mu \mathrm{m}$ square of freestanding membrane was formed by removing thin $\mathrm{SiN}$ and $\mathrm{SiO}_{2}$ film windows on one side using reactive ion etching (RIE), followed by $\mathrm{KOH}$ $\left(40 \%, 80^{\circ} \mathrm{C}\right)$ etching of the Si wafer. Then focused ion beam (FIB, DB235) was used to remove about $1.5 \mu \mathrm{m}$ thick silicon oxide with size of $1 \mu \mathrm{m}$ from the center of freestanding membrane. $2 \mu \mathrm{m}$ square free-standing membrane of silicon nitride was finally formed after the remaining $500 \mathrm{~nm}$ thick silicon oxide was etched by 6:1 buffered oxide etching (BOE). The nanopores were drilled in the center of silicon nitride membrane using a focused $300 \mathrm{kV}$ electron beam from TEM (Tecnai F30).

\section{Protein preparation}

Gp10 C-his affinity tag plasmids were constructed from vector pET-21a (+) (Novagen) with a two-step polymerase chain reaction (PCR). Then the plasmids constructed previously were transformed into the E.coli strain HMS174 (DE3) for protein expression. Inoculate $10 \mathrm{~mL} \mathrm{LB}$ broth and Ampicilin (50 $\mu \mathrm{g} \mathrm{mL} \mathrm{mL}^{-1}$ 1:1000 dilution) with frozen stock. $1-2 \mu \mathrm{L}$ bacterial were added to the liquid culture and then incubated overnight at $37^{\circ} \mathrm{C}, 180 \mathrm{rpm}$. On the sec- ond day, Incubate $500 \mathrm{~mL}$ broth with $10 \mathrm{~mL}$ of an overnight culture until the culture achieves an optical density of $0.4-0.6$. A final concentration of $0.4 \mathrm{mM}$ isopropyl $\beta$-D-1-thiogalactopyranoside (IPTG) was added into the cultured medium, which was incubated for another $3 \mathrm{~h}$. The cells were harvested by centrifugation at $6000 \mathrm{rpm}$ for $20 \mathrm{~min}$ in a Beckman JS-21 rotor, followed by French Press, and digested with DNase I and RNase A on ice for half an hour. Cell lysate was clarified by centrifugation and filtration. Then purification of C-His-tagged connector proteins was conducted with one-step immobilized metal affinity chromatography (IMAC). 2 mL Ni-NTA His-bind resin (Novagen, San Diego, CA) was added into a column and regenerated with $10 \mathrm{~mL}$ charge buffer (50 $\mathrm{mM} \mathrm{NiCl}_{2}$ ) and $10 \mathrm{~mL}$ His-wash buffer (15\% glycerol, $500 \mathrm{mM} \mathrm{NaCl}$, $50 \mathrm{mM}$ ATP, $50 \mathrm{mM}$ imidazole, and $100 \mathrm{mM}$ Tris- $\mathrm{HCl}, \mathrm{pH}$ 8.0). Cell pellets were resuspended in His-bind buffer (15\% glycerol, $500 \mathrm{mM} \mathrm{NaCl}, 50 \mathrm{mM}$ ATP, $10 \mathrm{mM}$ imidazole, and $100 \mathrm{mM}$ Tris-HCl, $\mathrm{pH}$ 8.0). Cell lysate was clarified by centrifugation at $12,500 \mathrm{rpm}, 4^{\circ} \mathrm{C}$ (Beckman $\mathrm{J} 2-21$, rotor JA-20) for $20 \mathrm{~min}$ and filtration followed by loading onto His-bind resin by gravity flow. $10 \mathrm{~mL}$ His-wash buffer was used to remove impurities. $5 \mathrm{~mL}$ aliquots of His-elution buffer (15\% glycerol, $500 \mathrm{mM} \mathrm{NaCl}, 50 \mathrm{mM}$ ATP, $500 \mathrm{mM}$ imidazole, and $100 \mathrm{mM}$ Tris- $\mathrm{HCl}, \mathrm{pH}$ 8.0) was applied to elute His-tagged gp10 protein. Then the sample was collected with every $0.5 \mathrm{~mL}$ elution coming out.

\section{Phi29 connector protein translocation measurements}

Each chip with a nanopore was rinsed by a standard process including ultrapure water, nitrogen gas and plasma cleaner, then sealed with polydimethylsiloxane (PDMS) gaskets which were later mounted between the polyether ether ketone (PEEK) chambers. The trans and cis chambers full of ionic solution ( $0.5 \mathrm{M} \mathrm{KCl}$ with $5 \mathrm{mM}$ Tris) were electrically contacted with $\mathrm{Ag} / \mathrm{AgCl}$ electrodes and bias voltage was applied by an Axon 200B patch clamp amplifier (Molecular Devices, Sunnyvale,CA) operating with an 8-pole, $40 \mathrm{kHz}$, low pass Bessel filter. Then the electrical measurement was conducted in a dark Faraday cage after the phi29 connector proteins were added in the cis chamber. Amplifier output was digitized at $250 \mathrm{kHz}$ and continuously recorded to a disk using an Axon Digidata 1440A digitizer and pClamp 10 software.

\section{Simulations}

Calculations of open current through the nanopore, current blockage of protein in the nanopore at different pore radius, and pore length were performed using finite-element numerical calculations of a Poisson-Boltzmann for- 
mulation, as previously described [46]. To determine the geometry parameters of the nanopore, we have done a series of ionic current calculations with and without the protein inside the nanopore respectively. Since the thickness of the nanopore membrane inside the nanopore is unchangeable, the only two parameters that determine the nanopore geometry are the pore radius and the effective length (the length of the cylindrical part of the nanopore channel). We swept a large variation in both these two parameters and compared the calculated current and blockage value with the experimental result until they matched up. In this way, the exact geometry parameters of the nanopore can be determined in experiment.

Received 19 May 2015; accepted 4 June 2015; published online 15 June 2015

1 Kasianowicz JJ, Brandin E, Branton D, et al. Characterization of individual polynucleotide molecules using a membrane channel. Proc Natl Acad Sci USA, 1996, 93: 13770-13773

2 Clarke J, Wu HC, Jayasinghe L, et al. Continuous base identification for single-molecule nanopore DNA sequencing. Nat Nanotechnol, 2009, 4: 265-270

3 Ying YL, Wang HY, Sutherland TC, et al. Monitoring of an ATPbinding aptamer and its conformational changes using an $\alpha$-hemolysin nanopore. Small, 2011, 7: 87-94

4 Ying YL, Zhang J, Gao R, et al. Nanopore-based sequencing and detection of nucleic acids. Angew Chem Int Ed, 2013, 52: $13154-$ 13161

5 Ying YL, Zhang J, Meng FN, et al. A stimuli-responsive nanopore based on a photoresponsive host-guest system. Sci Rep, 2013, 3: 1662

6 Li J, Stein D, McMullan C, et al. Ion-beam sculpting at nanometre length scales. Nature, 2001, 412: 166-169

7 Li J, Gershow M, Stein D, et al. DNA molecules and configurations in a solid-state nanopore microscope. Nat Mater, 2003, 2: 611-615

8 Dekker C. Solid-state nanopores. Nat Nanotechnol, 2007, 2: 209215

9 Wendell D, Jing P, Geng J, et al. Translocation of double-stranded DNA through membrane-adapted phi29 motor protein nanopores. Nat Nanotechnol, 2009, 4: 765-772

10 Garaj S, Hubbard W, Reina A, et al. Graphene as a subnanometre trans-electrode membrane. Nature, 2010, 467: 190-193

11 Geng J, Wang S, Fang H, et al. Channel size conversion of phi29 DNA-packaging nanomotor for discrimination of single-and double-stranded nucleic acids. ACS Nano, 2013, 7: 3315-3323

12 Deamer DW, Branton D. Characterization of nucleic acids by nanopore analysis. Acc Chem Res, 2002, 35: 817-825

13 Skinner GM, van den Hout M, Broekmans O, et al. Distinguishing single-and double-stranded nucleic acid molecules using solid-state nanopores. Nano Lett, 2009, 9: 2953-2960

14 Talaga DS, Li J. Single-molecule protein unfolding in solid state nanopores. J Am Chem Soc, 2009, 131: 9287-9297

15 Yusko EC, Johnson JM, Majd S, et al. Controlling protein translocation through nanopores with bio-inspired fluid walls. Nat Nanotechnol, 2011, 6: 253-260

16 Fologea D, Ledden B, McNabb DS, et al. Electrical characterization of protein molecules by a solid-state nanopore. App Phys Lett, 2007, 91: 053901

17 Li J, Fologea D, Rollings R, et al. Characterization of protein un- folding with solid-state nanopores. Protein Peptide Lett, 2014, 21: 256-265

18 Ledden B, Fologea D, Talaga DS, et al. Sensing Single Protein Molecules with Solid-state Nanopores. In: Iqbal SM, Bashir R (Eds.). Nanopores. Heidelberg: Springer, 2011, 129-150

19 Cressiot B, Oukhaled A, Patriarche G, et al. Protein transport through a narrow solid-state nanopore at high voltage: experiments and theory. ACS Nano, 2012, 6: 6236-6243

20 Movileanu L. Interrogating single proteins through nanopores: challenges and opportunities. Trends biotechnol, 2009, 27:333-341

21 Han A, Schürmann G, Mondin G, et al. Sensing protein molecules using nanofabricated pores. App Phys Lett, 2006, 88: 093901

22 Rotem D, Jayasinghe L, Salichou M, et al. Protein detection by nanopores equipped with aptamers. J Am Chem Soc, 2012, 134: 2781-2787

23 Sexton LT, Mukaibo H, Katira P, et al. An adsorption-based model for pulse duration in resistive-pulse protein sensing. J Am Chem Soc, 2010, 132: 6755-6763

24 Rodriguez-Larrea D, Bayley H. Multistep protein unfolding during nanopore translocation. Nat Nanotechnol, 2013, 8: 288-295

25 Winzor DJ, Jones S, Harding SE. Determination of protein charge by capillary zone electrophoresis. Anal Biochem, 2004, 333: 225-229

26 Freedman KJ, Haq SR, Edel JB, et al. Single molecule unfolding and stretching of protein domains inside a solid-state nanopore by electric field. Sci Rep, 2013, 3: 1638

27 Storm A, Chen J, Ling X, et al. Fabrication of solid-state nanopores with single-nanometre precision. Nat Mater, 2003, 2: 537-540

28 Wanunu M, Meller A. Chemically modified solid-state nanopores. Nano Lett, 2007, 7: 1580-1585

29 Squires AH, Hersey JS, Grinstaff MW, et al. A nanopore-nanofiber mesh biosensor to control DNA translocation. J Am Chem Soc, 2013, 135: 16304-16307

30 Fologea D, Gershow M, Ledden B, et al. Detecting single stranded DNA with a solid state nanopore. Nano Lett, 2005, 5: 1905-1909

31 Liu S, Lu B, Zhao Q, et al. Boron nitride nanopores: highly sensitive DNA single-molecule detectors. Adv Mater, 2013, 25: 4549-4554

32 Larkin J, Henley R, Bell DC, et al. Slow DNA transport through nanopores in hafnium oxide membranes. ACS Nano, 2013, 7: 10121-10128

33 Lu B, Hoogerheide DP, Zhao Q, et al. Pressure-controlled motion of single polymers through solid-state nanopores. Nano Lett, 2013, 13: 3048-3052

34 Tang Z, Lu B, Zhao Q, et al. Surface modification of solid-state nanopores for sticky-free translocation of single-stranded DNA. Small, 2014, 10: 4332-4339

35 Brewer SH, Glomm WR, Johnson MC, et al. Probing BSA binding to citrate-coated gold nanoparticles and surfaces. Langmuir, 2005, 21: 9303-9307

36 Norde W, Arai T, Shirahama H. Protein adsorption in model systems. Biofouling, 1991, 4: 37-51

37 Niedzwiecki DJ, Grazul J, Movileanu L. Single-molecule observation of protein adsorption onto an inorganic surface. J Am Chem Soc, 2010, 132: 10816-10822

38 Roach P, Farrar D, Perry CC. Interpretation of protein adsorption: surface-induced conformational changes. J Am Chem Soc, 2005, 127: 8168-8173

39 Roach P, Farrar D, Perry CC. Surface tailoring for controlled protein adsorption: effect of topography at the nanometer scale and chemistry. J Am Chem Soc, 2006, 128: 3939-3945

40 Haque F, Li J, Wu HC, et al. Solid-state and biological nanopore for real-time sensing of single chemical and sequencing of DNA. Nano Today, 2013, 8: 56-74

41 Lee TJ, Schwartz C, Guo P. Construction of bacteriophage phi29 
DNA packaging motor and its applications in nanotechnology and therapy. Ann Biomed Eng, 2009, 37: 2064-2081

42 Larkin J, Henley RY, Muthukumar M, et al. High-bandwidth protein analysis using solid-state nanopores. Biophys J, 2014, 106: 696-704

43 Plesa C, Kowalczyk SW, Zinsmeester R, et al. Fast translocation of proteins through solid state nanopores. Nano Lett, 2013, 13: 658663

44 Kramers HA. Brownian motion in a field of force and the diffusion model of chemical reactions. Physica, 1940, 7: 284-304

45 Fologea D, Uplinger J, Thomas B, et al. Slowing DNA translocation in a solid-state nanopore. Nano Lett, 2005, 5: 1734-1737

$46 \mathrm{Lu} \mathrm{B}$, Hoogerheide DP, Zhao Q, et al. Effective driving force applied on DNA inside a solid-state nanopore. Phys Rev E, 2012, 86: 011921

47 DeBlois R, Bean C. Counting and sizing of submicron particles by the resistive pulse technique. Rev Sci Instrum, 1970, 41: 909-916

48 Firnkes M, Pedone D, Knezevic J, et al. Electrically facilitated translocations of proteins through silicon nitride nanopores: conjoint and competitive action of diffusion, electrophoresis, and electroosmosis. Nano Lett, 2010, 10: 2162-2167

49 Han A, Creus M, Schürmann G, et al. Label-free detection of single protein molecules and protein-protein interactions using synthetic nanopores. Anal Chem, 2008, 80: 4651-4658
50 He Y, Tsutsui M, Fan C, et al. Controlling DNA translocation through gate modulation of nanopore wall surface charges. ACS Nano, 2011, 5: 5509-5518

51 Hoogerheide DP, Garaj S, Golovchenko JA. Probing surface charge fluctuations with solid-state nanopores. Phys Rev Lett, 2009, 102: 256804

Acknowledgements This work was supported by the National Basic Research Program from the Ministry of Science and Technology of China (2011CB707601 and 2013CB932602), and the National Natural Science Foundation of China (51272007, 91433102 and 11234001). Zhao Q acknowledges Beijing Nova Program (XX2013003) and the Program for New Century Excellent Talents in University of China

Author contributions Guo P and Zhao Q designed the experiments. Zhao Q and Yu D supervised and directed the projects. Li J performed the experiments and data analysis. Fu Q helped to make the nanopores. Li J and Zhao Q wrote the paper. Yan E and Wang S engineered the protein samples; Tang $\mathrm{Z}$ and Hu R contributed to the theoretical analysis. All authors contributed to the general discussion.

Conflict of interest The authors declare that they have no conflict of interest. 


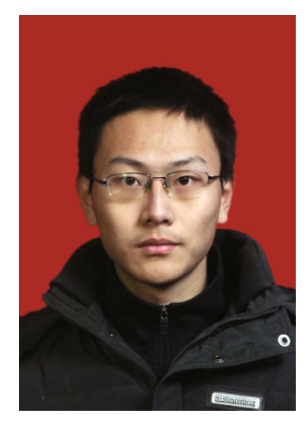

Ji Li graduated from Shandong University in 2012 majored in physics. He is currently pursuing his $\mathrm{PhD}$ degree in the field of single-molecule detection and improvement of temporal and spatial resolution with solid-state nanopore in the group of Prof. Zhao and Prof. Yu.

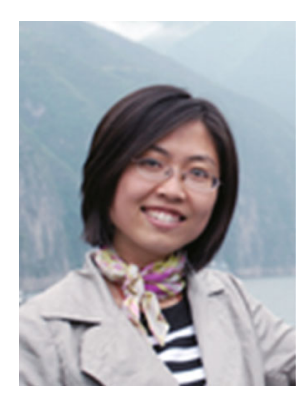

Qing Zhao received her PhD degree in physics from Peking University in 2006. After her postdoctoral research at University of Washington, she joined the School of Physics, Peking University as an associate professor. Her research focuses on single molecule detection based on solid-state nanopores and low dimensional photovoltaic energy devices. She has published more than 50 SCI papers which have been cited more than 1500 times with an h-index of 21 (2014).

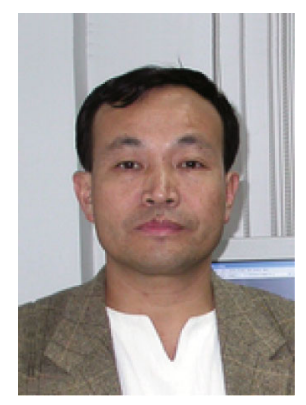

Dapeng Yu is a professor of the School of Physics at Peking University. He was awarded the "National Outstanding Youth Science Fund" in 2000 and awarded Changjiang Scholars by the Ministry of Education of China in 2002. His recent research focuses on low dimensional physics and nanostrutres, including quantum transport of 2D materials, semiconductor nanowires, nanopore biosensors and energy devices.

中文摘要 纳米孔是一种有效的探测蛋白质以及蛋白质复合物的工具. 蛋白质与纳米孔的相互作用是生物和生物医学领域普遍存在的 基础过程. 本文通过分析phi29蛋白穿过氮化硅纳米孔产生的电信号, 初步实现了单分子层面对蛋白疏水表面的探测. phi29蛋白穿孔过 程中独特的“双层事件”以及双层事件深度的比例表明相互作用发生在phi29蛋白的疏水表面. 本文提供了一种在蛋白表面定位疏水区 域的潜在方法, 揭示了疏水相互作用在蛋白和纳米孔的相互作用中扮演着重要的角色. 另外, 该研究在固态纳米孔探测蛋白表面化学 性质等领域也具有巨大的潜在意义. 